\title{
BREAST CANCER IN WOMEN: CHARACTERIZATION OF CASES INCLUDED IN THE HOSPITAL-BASED CANCER REGISTRY OF THE STATE OF SÃO PAULO AND FACTORS ASSOCIATED WITH ADVANCED STAGES
}

Raissa Janine de Almeidaㄹ, Arthur Felipe Decker ${ }^{1}$, Carolina Terra de Moraes Luizaga², Cristiane Murta-Nascimento ${ }^{1}$ Faculdade de Medicina de Botucatu, Universidade Estadual Paulista - Botucatu (SP), Brazil.

${ }^{2}$ Fundação Oncocentro de São Paulo - São Paulo (SP), Brazil.

Introduction: Breast cancer is a public health problem worldwide, being the most common malignancy in the female population. It is a potentially curable disease with an early diagnosis. The advanced stage at the time of diagnosis is associated with increased morbidity and low survival for the patients. Objectives: To describe the sociodemographic, clinical, and anatomopathological characteristics of breast cancer cases in women included in the hospital-based cancer registry (RHC) of Fundação Oncocentro de São Paulo (FOSP), and to investigate factors associated with the clinical stage at diagnosis. Methods: The study design was a case series. The sample consisted of women with breast cancer diagnosed between 2000 and 2014 and included in the RHC-FOSP. The outcome variable was a clinical-stage (stage 0-II versus III-IV). The explanatory variables were age at diagnosis and educational level. This study was approved by the Human Research Ethics Committee of the Botucatu Medical School, São Paulo State University, Brazil. Results: The study included 84,987 women with in situ and invasive breast cancer diagnosed between 2000 and 2014. The average age of women at diagnosis was 56.7 years (95\%CI 56.6-56.8 years). Sixty-five percent of cases completed elementary school or less and the most frequent histological type was ductal carcinoma (77.2\%). During the study period, there was a slight decrease in the proportion of tumors in more advanced stages, from $39.8 \%$ in 2000 to $32.6 \%$ in 2014 . There was a statistically significant association between age at diagnosis and educational level with the women's grouped clinical stage. Women of older age and those with a higher education level had reduced odds ratios of having tumors in more advanced stages at the time of diagnosis. Conclusions: These findings may contribute to the development of policies for the identification of breast tumors at earlier stages. 